\title{
Response of mean arterial pressure to temporary biventricular pacing after chest closure during cardiac surgery
}

\author{
Benjamin J. Rubinstein, BA, ${ }^{a}$ Daniel Y. Wang, MD, ${ }^{\mathrm{b}}$ Santos E. Cabreriza, MBA, ${ }^{\mathrm{c}}$ Bin Cheng, PhD, ${ }^{\mathrm{d}}$ \\ Linda Aponte-Patel, MD, ${ }^{\mathrm{e}}$ Alexandra Murata, BA, ${ }^{\mathrm{c}}$ Alexander Rusanov, MD, ${ }^{\mathrm{f}}$ Marc E. Richmond, MD, \\ T. Alexander Quinn, $\mathrm{PhD},{ }^{\mathrm{g}, \mathrm{h}}$ and Henry M. Spotnitz, $\mathrm{MD}^{\mathrm{c}}$
}

\begin{abstract}
Objectives: We have previously demonstrated that biventricular pacing increased cardiac output within 1 hour of weaning from cardiopulmonary bypass in selected patients. To assess the possible sustained benefit, we reviewed in the present study the effects of biventricular pacing on the mean arterial pressure after chest closure.

Methods: A total of 30 patients (mean ejection fraction 35\% $\pm 15 \%$, mean QRS $119 \pm 24$ ms) underwent coronary bypass and/or valve surgery. The mean arterial pressure was maximized during biventricular pacing using atrioventricular delays of 90 to $270 \mathrm{~ms}$ and interventricular delays of +80 to $-80 \mathrm{~ms}$ during 20 -second intervals in random sequence. Optimized biventricular pacing was finally compared with atrial pacing at a matched heart rate and to a sinus rhythm during 30-second intervals. Vasoactive medication and fluid infusion rates were held constant. The arterial pressure was digitized, recorded, and integrated. Statistical significance was assessed using linear mixed effects models and Bonferroni's correction.
\end{abstract}

Results: Optimized atrioventricular delay, ranging from 90 to $270 \mathrm{~ms}$, increased the mean arterial pressure 4\% versus nominal and $7 \%$ versus the worst $(P<.001)$. Optimized interventricular delay increased pressure 3\% versus nominal and $7 \%$ versus the worst. Optimized biventricular pacing increased the mean arterial pressure $4 \%$ versus sinus rhythm $(78.5 \pm 2.4$ vs $75.1 \pm 2.4 \mathrm{~mm} \mathrm{Hg} ; P=.002)$ and $3 \%$ versus atrial pacing $(76.4 \pm 2.7$ $\mathrm{mm} \mathrm{Hg} ; P=.017)$.

Conclusions: Temporary biventricular pacing improves the hemodynamics after chest closure, with effects similar to those within 1 hour of bypass. Individualized optimization of atrioventricular delay is warranted, because the optimal delay was longer in $80 \%$ of our patients than the current recommendations for temporary postoperative pacing. (J Thorac Cardiovasc Surg 2012;144:1445-52)

Cardiac resynchronization therapy, or biventricular pacing (BiVP), has been proven efficacious for the treatment of chronic heart failure, although its use in acute conditions is less well established. Currently, permanent BiVP is the standard of care for patients with medication-refractory severe heart failure with left ventricular (LV) dysfunction and intraventricular conduction delay. ${ }^{1}$ BiVP reduces the

\footnotetext{
From the Columbia University College of Physicians and Surgeons, ${ }^{a}$ New York, NY; Departments of Medicine, ${ }^{\mathrm{b}}$ Surgery, ${ }^{\mathrm{c}}$ Pediatrics, ${ }^{\mathrm{e}}$ and Anesthesiology,${ }^{\mathrm{f}}$ Columbia University, New York, NY; Department of Biostatistics, ${ }^{\mathrm{d}}$ Columbia University Mailman School of Public Health, New York, NY; National Heart and Lung Institute, ${ }^{\mathrm{g}}$ Imperial College London, Harefield, United Kingdom; and Department of Computer Science, ${ }^{\text {h }}$ University of Oxford, Oxford, United Kingdom.

This study was funded by a grant from the National Institutes of Health (grant RO1 HL080152 to H.M.S.); Dr Wang was supported by National Institutes of Health Training Grant T32 HL007854; and Dr Rusanov was supported by National Institutes of Health Training Grant 5 T32 GM008464-17.

Disclosures: Dr Spotnitz is on the Scientific Advisory Board of, and holds an ownership interest in, Biophan Technologies, Inc; the remaining authors have nothing to disclose with regard to commercial support.

Dr Spotnitz is the George H. Humphreys II Professor of Surgery.

Received for publication Jan 10, 2012; revisions received March 6, 2012; accepted for publication April 4, 2012; available ahead of print Aug 23, 2012

Address for reprints: Henry M. Spotnitz, MD, Department of Surgery, Columbia University Medical Center, 622 West 168th St, 10th Floor, Vanderbilt Clinic, Room 1010, New York, NY (E-mail: hms2@columbia.edu).

$0022-5223 / \$ 36.00$

Copyright (c) 2012 by The American Association for Thoracic Surgery

http://dx.doi.org/10.1016/j.jtcvs.2012.04.026
}

morbidity and mortality in this population and improves the LV geometry and function. ${ }^{2-5}$ BiVP is effective even in patients with mildly symptomatic (New York Heart Association class I and II) heart failure with a low ejection fraction and intraventricular conduction delay, reducing heart failure events and increasing the interval to the first hospitalization. ${ }^{6,7}$

Although BiVP is clinically useful, more than $30 \%$ of implants do not result in objective or subjective benefits. ${ }^{3}$ Programmable parameters that can be manipulated to optimize BiVP include atrioventricular delay (AVD) and interventricular delay (VVD). The hemodynamic indexes used to assess optimization include cardiac output (CO) and mean arterial pressure (MAP) ${ }^{8-10}$

The effects of temporary BiVP in low output states after cardiac surgery have been previously studied. ${ }^{7}$ Investigation has centered on patients with a low LV ejection fraction (LVEF) preoperatively, because this measure independently predicts the risk of acute heart failure. ${ }^{11}$ The clinical results of these studies have been mixed, with the greatest benefit observed early after cardiopulmonary bypass $(\mathrm{CPB}){ }^{12,13} \mathrm{BiVP}$ is a promising adjunct to pharmacologic support after surgery, because it can improve the stroke volume without increasing myocardial oxygen consumption. ${ }^{14}$ Patient selection and 


$$
\begin{aligned}
& \text { Abbreviations and Acronyms } \\
& \begin{aligned}
\text { AAI } & =\text { atrial pacing } \\
\text { AVD } & =\text { atrioventricular delay } \\
\text { BIPACS } & =\text { Biventricular Pacing After Cardiac } \\
& \text { Surgery } \\
\text { BiVP } & =\text { biventricular pacing } \\
\mathrm{CO} & =\text { cardiac output } \\
\mathrm{CPB} & =\text { cardiopulmonary bypass } \\
\mathrm{LV} & =\text { left ventricular } \\
\mathrm{LVEF} & =\mathrm{LV} \text { ejection fraction } \\
\text { MAP } & =\text { mean arterial pressure } \\
\text { VVD } & =\text { interventricular delay }
\end{aligned}
\end{aligned}
$$

the optimal pacing protocols in this setting have not yet been defined.

The Biventricular Pacing After Cardiac Surgery (BIPACS) trial is a randomized clinical trial evaluating the use of an optimized pacing protocol, including BiVP perioperatively in patients with a low preoperative LVEF and an intraventricular conduction delay. The clinical trial registration number was NCT00498940 (http://ClinicalTrials.gov). The primary hypothesis was that continuous optimized pacing will increase the $\mathrm{CO}$ $15 \%$ compared with the standard of care. Pacing is optimized at 3 points. Phase I is initiated within 1 hour of weaning from $\mathrm{CPB}$, phase II at the conclusion of surgery, and phase III 12 to 24 hours later. The phase I substudy analysis showed that optimization of AVD and VVD independently increased the $\mathrm{CO}$, as measured by an aortic flow probe. This was assessed in all patients in the BiVP and control groups, comparing the effects within each patient rather than between the patient groups. Optimized BiVP increased the CO $10 \%$ compared with sinus rhythm and $13 \%$ compared with atrial pacing at the same rate, indicating that the hemodynamic benefit was independent of the increased heart rate. ${ }^{15}$ However, the preliminary data from our own group, as well as others, have suggested that the BiVP effects might not be sustained over time after CPB. Accordingly, we initiated the present study using data from phase II, after chest closure. In the absence of direct measures of $\mathrm{CO}$, the MAP was used as the optimization marker. We hypothesized that BiVP optimization would increase the MAP and that optimized BiVP would increase the MAP compared with sinus rhythm or atrial pacing.

\section{METHODS \\ BiPACS Study Population}

The Columbia University Medical Center institutional review board approved the study protocol, and the study was conducted under support from the National Institutes of Health with an investigational device exemption from the Food and Drug Administration. Adult patients undergoing elective open heart surgery with CPB are screened for eligibility to enroll in the BiPACS trial. All patients provided written, informed consent. The inclusion criteria were preoperative congestive heart failure, LVEF of $40 \%$ or less, and QRS duration of $100 \mathrm{~ms}$ or more, or patients undergoing combined mitral and aortic valve surgery. The LVEF and QRS criterion was liberalized from $35 \%$ and $120 \mathrm{~ms}$, respectively, in the original protocol. The exclusion criteria were atrial fibrillation, second- or third-degree atrioventricular block, congenital heart disease, intracardiac shunt, or heart rate greater than $120 \mathrm{bpm}$ after separation from CPB. The preoperative data obtained by chart review included LVEF, as measured on the echocardiogram or left ventriculogram; heart rhythm, QRS duration, and intraventricular block from the electrocardiographic tracings; type of surgery performed; and demographic characteristics. The BiPACS trial is ongoing and the study target 206 patients. The study is currently being expanded to the University of California at Los Angeles.

\section{Study Design and Optimization Protocol}

Patients in the BiPACS trial are randomized to 2 treatment groups at the end of phase I. To avoid imbalances that can occur using simple randomization, randomly permuted blocks of 4,6 , and 8 are used with a treatment allocation ratio of one, with each group to be of equal size. The phase II testing described in the present report is performed in all patients completing phase I and randomized. AVD is optimized first, followed by the ventricular pacing site, and then VVD. The settings for each individual optimization parameter are tested in random sequence. The detailed methods of the protocol have been previously reported. ${ }^{15}$ Phase I optimization and testing occur after stabilizing vasoactive drug and volume requirements post-CPB. Standard hemodynamic monitoring, including systemic and pulmonary arterial pressures and transesophageal echocardiography, are used. Patients completing phase I are then randomized to continuous BiVP or the standard of care. BiVP patients are paced using optimized phase I settings until phase II. After closure of the chest and establishment of stable inotrope and vasopressor dosing, the phase II BiVP optimization protocol is initiated. The pacing leads are attached to a Medtronic InSync III permanent biventricular pacemaker (Medtronic, Inc, Minneapolis, Minn) mounted in an external housing unit, and their sensing and pacing functions are tested and confirmed. The use of this pacemaker for temporary BiVP is under an investigational device exemption from the Food and Drug Administration. The pacing rate is $90 \mathrm{bpm}$, or $10 \mathrm{bpm}$ greater than the patient's intrinsic heart rate if greater than $90 \mathrm{bpm}$ to ensure atrial capture, up to a maximum of $120 \mathrm{bpm}$. The electrocardiographic and arterial pressure signals are collected with an analog-todigital converter (PowerLab, ADInstruments, Inc, Milford, Mass) and recorded on a personal computer (iMac, Apple Computer, Inc, Cupertino, Calif). The MAP is measured by averaging the digitized arterial pressure waveforms for 1 respiratory cycle using MacLab software (ADInstruments, Inc) and custom-designed routines in Matlab (MathWorks, Inc, Natick, Mass).

BiVP optimization is performed by optimization of AVD, followed by VVD. All pacing settings during optimization are conducted during 20 -second intervals and tested twice. Rapid optimization of the cardiac mechanics over brief intervals has been previously described. ${ }^{8,16,17}$ Determination of the interatrial delay (atrioventricular conduction prolongation) was formalized as follows. The intrinsic atrioventricular interval between atrial and ventricular sensing, with the pacemaker set to the dual-sensed (ODO) mode, is measured first, using data displayed on the programmer screen. Next, the interval between the atrial pacing impulse and the sensed ventricular response (paced atrioventricular interval) is determined, with the pacemaker set to the atrial paced-dual sensed mode (ADI). The atrioventricular conduction prolongation was determined using the formula, atrioventricular conduction prolongation = paced atrioventricular interval minus intrinsic atrioventricular interval, and was used to eliminate any inappropriate AVDs.

AVD optimization was performed during sequential pacing, with a VVD of $0 \mathrm{~ms}$. The AVD was varied in 30-ms increments (range, 
TABLE 1. Baseline clinical characteristics

\begin{tabular}{lc}
\hline Patients (n) & \\
Total & 30 \\
Optimization analysis & 30 \\
Optimized BiVP vs AAI vs sinus rhythm & 21 \\
Age (y) & $67 \pm 12$ \\
LVEF (\%) & $35 \pm 15$ \\
QRS duration (ms) & $119 \pm 24$ \\
Male gender (\%) & 77 \\
Surgery type (n) & \\
CABG/AVR, CABG/MVR, CABG/AVR/MVR & 13 \\
AVR/MVR & 8 \\
CABG & 6 \\
AVR & 3 \\
\hline
\end{tabular}

$B i V P$, Biventricular pacing; $A A I$, atrial pacing; $L V E F$, left ventricular ejection fraction; $C A B G$, coronary artery bypass grafting; $A V R$, aortic valve replacement; $M V R$, mitral valve replacement or repair.

90-270), in randomized sequence. AVDs longer than the patient's intrinsic paced AVD were not tested. Based on the average of 2 runs, the AVD yielding the greatest MAP was selected as the optimal AVD. VVD optimization was performed next using the optimal AVD and varying the VVD in 20-ms increments, ranging from $-80 \mathrm{~ms}$ (left ventricle first) to $+80 \mathrm{~ms}$ (right ventricle first), in randomized order. The MAP, averaged between 2 runs as a function of VVD, was plotted, and the VVD yielding the greatest MAP was selected as the optimal VVD. These steps defined the optimal BIVP protocol for each patient. The phase II optimized BiVP was then compared with the phase I BiVP settings, right atrial pacing (AAI mode) at the same heart rate, and to normal sinus rhythm (NSR) with no pacing, in randomized order, during 30-second intervals. The superior between the phase I BiVP settings and phase II optimized BiVP was selected as optimum and used for continuous pacing in the BiVP study group, pending additional optimization studies the next day.

The BiPACS trial uses the $\mathrm{CO}$ as the primary endpoint, predicated on the notion that organ flow is the primary determinant of morbidity and survival after cardiac surgery. We have also demonstrated that optimization of AVD and VVD during BiVP is based on the "dose-response curves," in which the changes in CO could be less than $5 \%$ for successive settings. Optimally, then, optimization should be based on serial changes in the $\mathrm{CO}$ that can be rapidly and accurately determined for very small changes. Optimization using our protocol involves measurement of $\mathrm{CO}$ for 38 pacemaker settings. In phase 1 , early after CPB, a very narrow window and greater requirement for accuracy for optimization favors flow probe use. ${ }^{15}$ Accuracy and speed are critical. However, the flow probe must be removed before chest closure. Thus, we chose the MAP as a surrogate for $\mathrm{CO}$ after chest closure. Thermal dilution methods were available as an alternative, because all patients had a pulmonary artery catheter placed; however, the long response time and extra volume administration for 38 measurements was considered prohibitive for a measurement with limited accuracy. The endpoint of the study in phase III, in the intensive care unit, does make use of thermal dilution, because slower measuring techniques are appropriate.

\section{Statistical Analysis}

For AVD and VVD optimization data and for the comparison among the optimized BiVP, AAI, and NSR, descriptive statistics were calculated for each group. Differences among multiple groups ( 3 groups) were tested

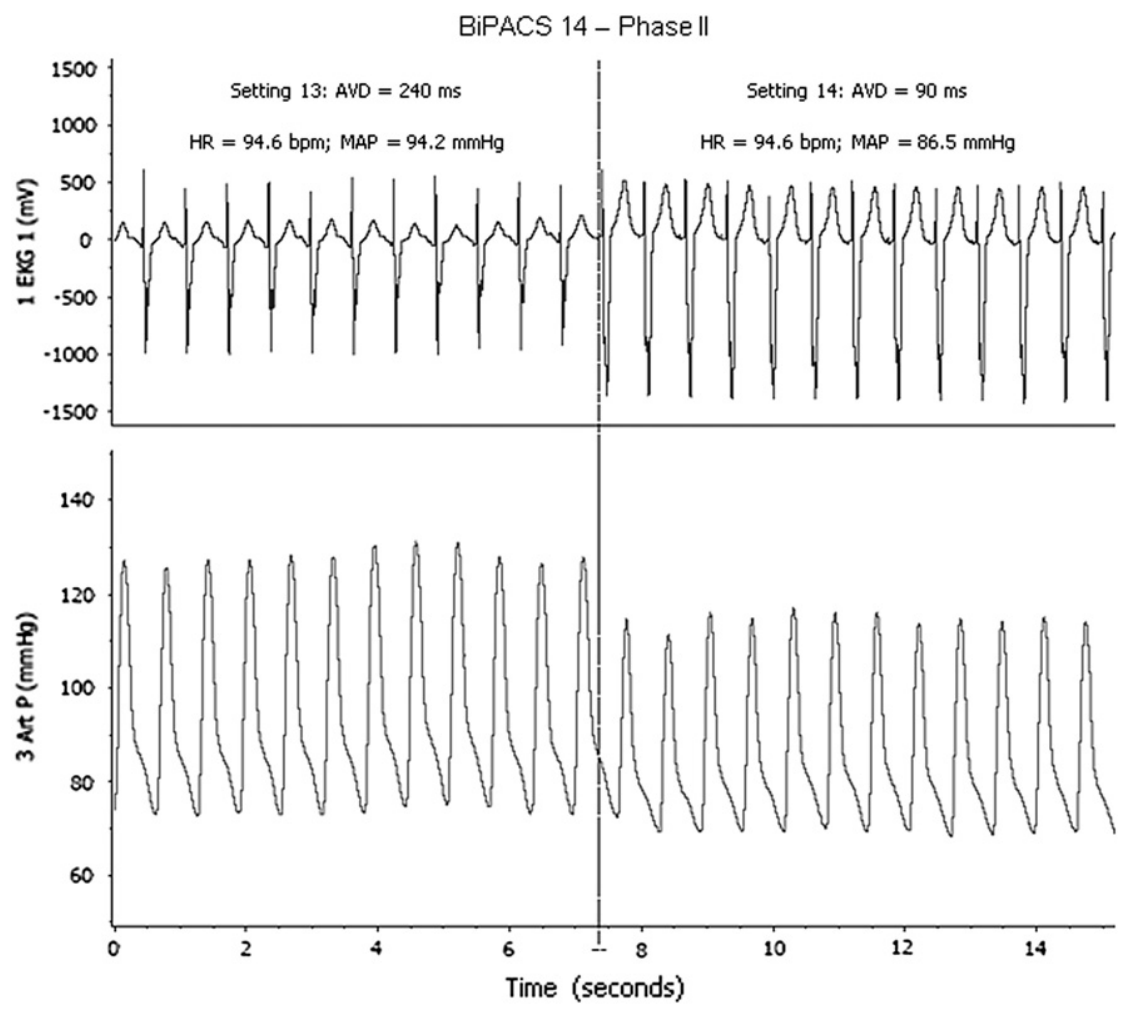

FIGURE 1. Representative intraoperative recording demonstrating changes in electrocardiogram $(E C G)$ and arterial pressure $(A r t P)$ when atrioventricular delay $(A V D)$ decreased from 240 to $90 \mathrm{~ms}$ in patient 14. Mean arterial pressure (MAP) decreased immediately from 94 to $86 \mathrm{~mm} \mathrm{Hg}$. This change reflected sequential implementation, by chance, of best and worst AVDs for this patient. A similar range of arterial pressures is shown in Figure 2 , A, for Patient 8 . BiPACS, Biventricular Pacing After Cardiac Surgery; HR, heart rate. 
using a linear mixed effects model. Post hoc comparisons to assess pairwise differences between groups were performed using the paired Student $t$ test. Bonferroni's correction was used to adjust for multiple comparisons. The distribution of the optimal AVD (or VVD) settings was analyzed to determine whether it differed from the default setting. Statistical analysis was performed using SAS, version 9.1 (SAS Institute, Inc, Cary, NC).

\section{RESULTS \\ Study Population}

The data were acquired in phase II for 44 patients of the 89 enrolled during the recruitment period (April 2007 to June 2010). BIVP optimization analysis was performed for 30 patients, with 14 excluded because of frequent ventricular ectopy, an intra-aortic balloon pump, or other factors affecting the arterial pressure data quality. Nine additional patients were excluded from the final comparison of the optimized BIVP, AAI, and NSR because of atrioventricular block and other factors, limiting the final comparison group to 21 patients. The baseline clinical characteristics are listed in Table 1. The demographic data were age, $67 \pm 12$ years, LVEF $35 \% \pm 15 \%$, and QRS duration $119 \pm 24 \mathrm{~ms}$ (mean $\pm \mathrm{SD}$ ). The proportion of male patients was $77 \%$. Most underwent valve surgery, either as a double/triple valve case (8 patients) or combined valve and coronary artery bypass grafting (13 patients); 6 underwent isolated coronary artery bypass grafting and 3 underwent isolated aortic surgery.

\section{Clinical Results}

A representative example of the immediate effects of the transition between 2 AVDs is shown in Figure 1, where a change in AVD from $240 \mathrm{~ms}$ to $90 \mathrm{~ms}$ resulted in an abrupt decrease in arterial pressure. Representative examples of AVD and VVD optimization are shown in Figure 2, $A, B$. The MAP was averaged over 2 runs and plotted to display the optimal pacing setting for each patient. For the patient represented in Figure 2, an AVD of $240 \mathrm{~ms}$, resulting in a peak MAP of $105.4 \mathrm{~mm} \mathrm{Hg}$, and a VVD of $+60 \mathrm{~ms}$ (right ventricle first), resulting in a peak MAP of $97.0 \mathrm{~mm} \mathrm{Hg}$, were optimal.

A comparison of the MAP for the optimized, worst, and nominal (120 ms) AVD settings showed significant differences among the groups $(P<.0001)$. In pairwise comparisons, the MAP was significantly different in the optimal and worst groups compared with that of the nominal AVD $(P<.001$ for both comparisons). The mean increase in the MAP from the worst AVD to the optimal AVD was $7 \%$ (range, $1 \%-18 \%$ ). The mean increase in the MAP from the nominal to the optimal AVD was $4 \%$ (range, $0 \%-11 \%$ ).

Differences in the MAP were significant comparing the optimal, worst, and nominal $(0 \mathrm{~ms})$ VVD settings $(P<.0001)$. In pairwise comparisons, both the optimal and worst VVD differed from the nominal VVD $(P<.0001$ and $P=.0005$, respectively). The mean increase in the MAP from the worst VVD to the optimal VVD was $7 \%$ (range, $1 \%-31 \%$ ). The mean increase in the MAP from the nominal to the optimal VVD was $4 \%$ (range, $0 \%-11 \%$ ).

The distribution of optimal AVDs is shown in Figure 3. An AVD of 90 ms was the optimal setting in only 2 patients. The optimum AVD was $120 \mathrm{~ms}$ in 4 patients and was 150 ms or more in 24 patients. The chance of the nominal setting of $120 \mathrm{~ms}$ being optimal was $13 \%$ (95\% confidence interval, 4\%-28\%) and the chance of the optimal setting being longer than $120 \mathrm{~ms}$ was $80 \%$ (95\% confidence interval, $64 \%-91 \%$ ). This justified the need for individualized optimization in AVD.

The distribution of optimal VVDs (Figure 4) was fairly uniform over the settings, with no clear preference of $\mathrm{RV}$-first versus LV-first pacing. The nominal VVD yielded the lowest MAP for 5 patients, and the chance of the default setting being optimal was $17 \%$ ( $95 \%$ confidence
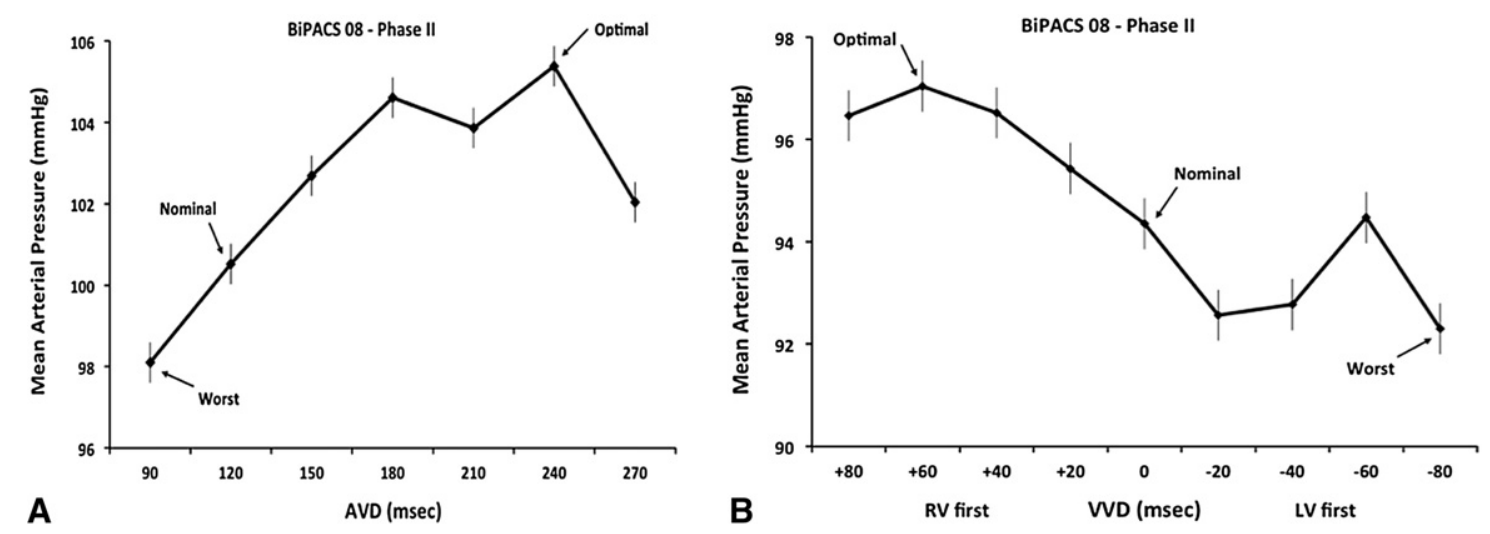

FIGURE 2. Representative example of effects of atrioventricular delay ( $A V D)$ and interventricular delay (VVD) optimization on mean arterial pressure $(M A P)$. Standard errors of averaged duplicate determinations shown in brackets. A, Best AVD according to MAP was 240 ms. Worst and nominal AVDs according to MAP are labeled. B, Best, worst, and nominal VVDs according to MAP are labeled. BiPACS, Biventricular Pacing After Cardiac Surgery; $R V$, right ventricle; $L V$, left ventricle; $V V D$, interventricular delay. 


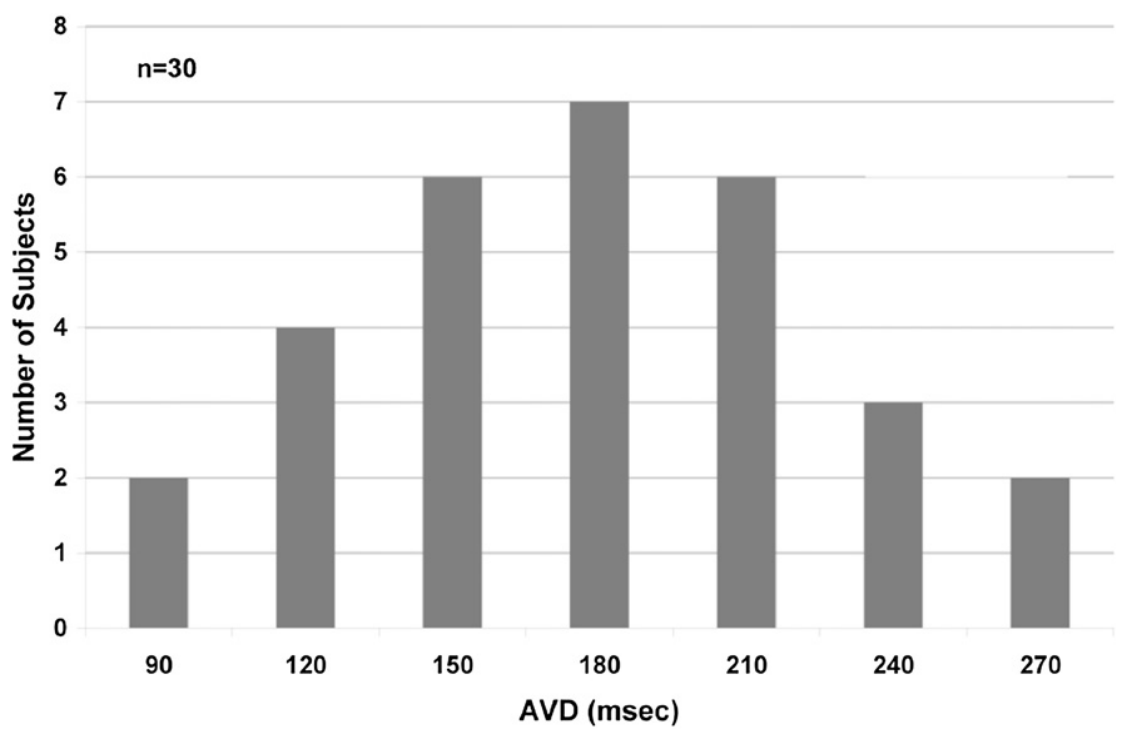

FIGURE 3. Optimized atrioventricular delay $(A V D)$ in 30 patients formed roughly normal distribution around median of $180 \mathrm{~ms}$.

interval, $6 \%-32 \%$ ), indicating the need for individualized optimization in VVD.

A comparison of the hemodynamics of optimized BiVP, AAI, and NSR is shown in Figure 5. The differences in the MAP among the 3 groups were significant $(P=.0002)$, as were the pairwise comparisons between the optimized BiVP, AAI, and NSR $(P=.0036$ and $P=.0006$, respectively). The optimized BiVP resulted in an increase in the MAP by $3 \%$ versus AAI at the same heart rate (MAP, $78.9 \pm 2.5$ vs $76.4 \pm 2.7 \mathrm{~mm} \mathrm{Hg}$, mean $\pm \mathrm{SEM}$ ) and by $5 \%$ versus NSR $(75.1 \pm 2.4 \mathrm{~mm} \mathrm{Hg})$. The increase in the MAP corresponded with a significant increase in the mean diastolic blood pressure by $3 \%$ versus AAI $(63.8 \pm$ 2.3 vs $62.2 \pm 2.4 \mathrm{~mm} \mathrm{Hg}$ ) and by $6 \%$ versus NSR (60.3 $\pm 2.3 \mathrm{~mm} \mathrm{Hg} ; P=.0043$ and $P=.0007$, respectively). The systolic blood pressure was unique, because it increased by $4 \%$ versus AAI $(108.8 \pm 3.4$ vs $104.5 \pm 3.6$ $\mathrm{mm} \mathrm{Hg} ; P=.0048)$; however, no significant difference was found compared with the NSR $(105.8 \pm 3.1 \mathrm{~mm} \mathrm{Hg})$. This pattern was reflected in the increased pulse pressure in BiVP by $6 \%$ versus AAI $(45.1 \pm 2.3$ vs $42.3 \pm 2.1$ $\mathrm{mm} \mathrm{Hg})$ nearing significance $(P=.0084)$ and no significant difference compared with NSR $(45.6 \pm 2.0 \mathrm{~mm} \mathrm{Hg})$. AAI resulted in a decrease in pulse pressure of $7 \%$ versus

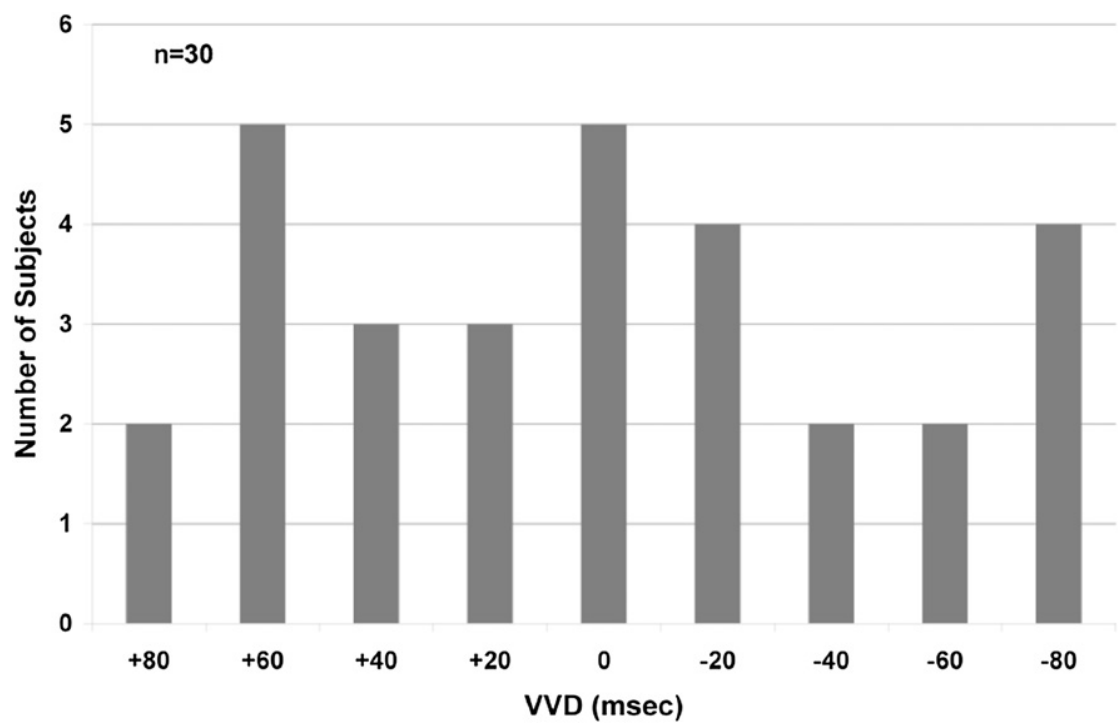

FIGURE 4. Optimized interventricular delay $(V V D)$ evenly scattered over full range of values explored in 30 patients. 


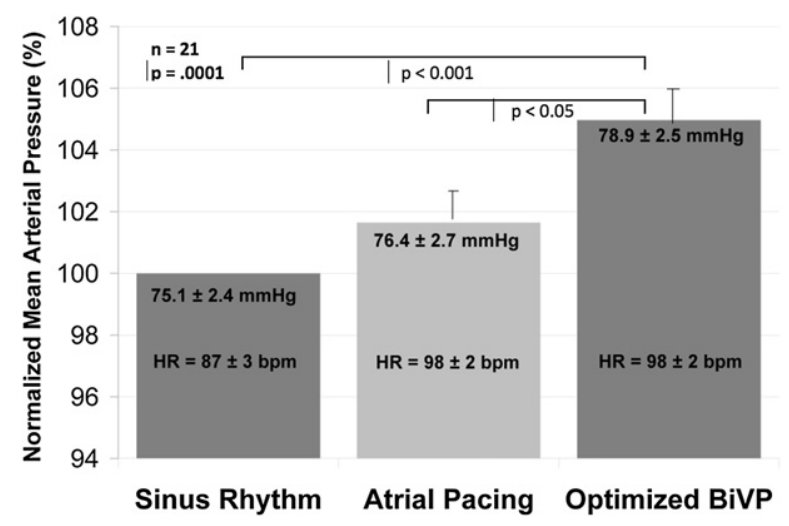

FIGURE 5. Average effect of optimized biventricular pacing (BiVP), atrial pacing $(A A I)$, and sinus rhythm (NSR) on normalized mean arterial pressure (MAP) in 30 patients. Error bars depict 1 standard error of mean. Differences were statistically significant. Absolute values for MAP and heart rate indicated in bars. $H R$, Heart rate.

NSR $(P=.0008)$. The paced heart rate was greater in the BiVP and AAI groups ( $98 \pm 2 \mathrm{bpm}$; mean \pm SEM) compared with the NSR group $(85 \pm 3 \mathrm{bpm} ; P<.0001)$.

\section{DISCUSSION}

A previous substudy from the BIPACS trial demonstrated that the $\mathrm{CO}$ increased during biventricular pacing versus both AAI and NSR within 1 hour of the conclusion of CPB. However, the benefits of perioperative pacing can change over time, and the optimal pacing protocol could also change. ${ }^{18,19}$ Accordingly, the present substudy was undertaken to determine whether hemodynamic benefit continues after chest closure. Because the aortic flow probe was not available in phase II, the hemodynamic index evaluated was the MAP. The present results have confirmed the statistically significant increases in MAP during biventricular pacing versus both AAI and NSR during phase II. Furthermore, optimization of both AVD and VVD independently contributed to hemodynamic benefit.

The mechanisms and techniques of BiVP optimization are of interest because more than $30 \%$ of recipients do not benefit from BiVP implants. For temporary pacing, our finding that MAP is useful for optimization is clinically relevant, because MAP is a preferred hemodynamic index for anesthesiologists.

Our results have also defined the optimal AVDs and VVDs, which were far from the common default values. AVD affects ventricular filling and mitral regurgitation. ${ }^{20}$ At greater heart rates, AVD optimization gains importance because of encroachment on the filling time. ${ }^{8}$ In the dynamic state of recovery from myocardial edema and inflammation after cardiac surgery, default values of 120 to $150 \mathrm{~ms}$ might be too short for diastolic filling. Our optimization data have demonstrated that the MAP increases $7 \%$ over the worst settings and $4 \%$ over the default, which translates roughly to a $14 \%$ and $8 \%$ change in the $\mathrm{CO}$, respectively. ${ }^{21}$ Prolongation of the interatrial and atrioventricular conduction times in the early postoperative period contributes to the need for long AVDs.

VVD optimization can improve hemodynamics by reducing the ventricular dyssynchrony associated with cardiomyopathy and might potentially compensate for a suboptimal lead position. ${ }^{4,22,23}$ In this substudy, VVD optimization using an optimal AVD increased MAP by an additional $7 \%$ compared with the worst setting and $3 \%$ compared with the default. In the case of the 5 patients
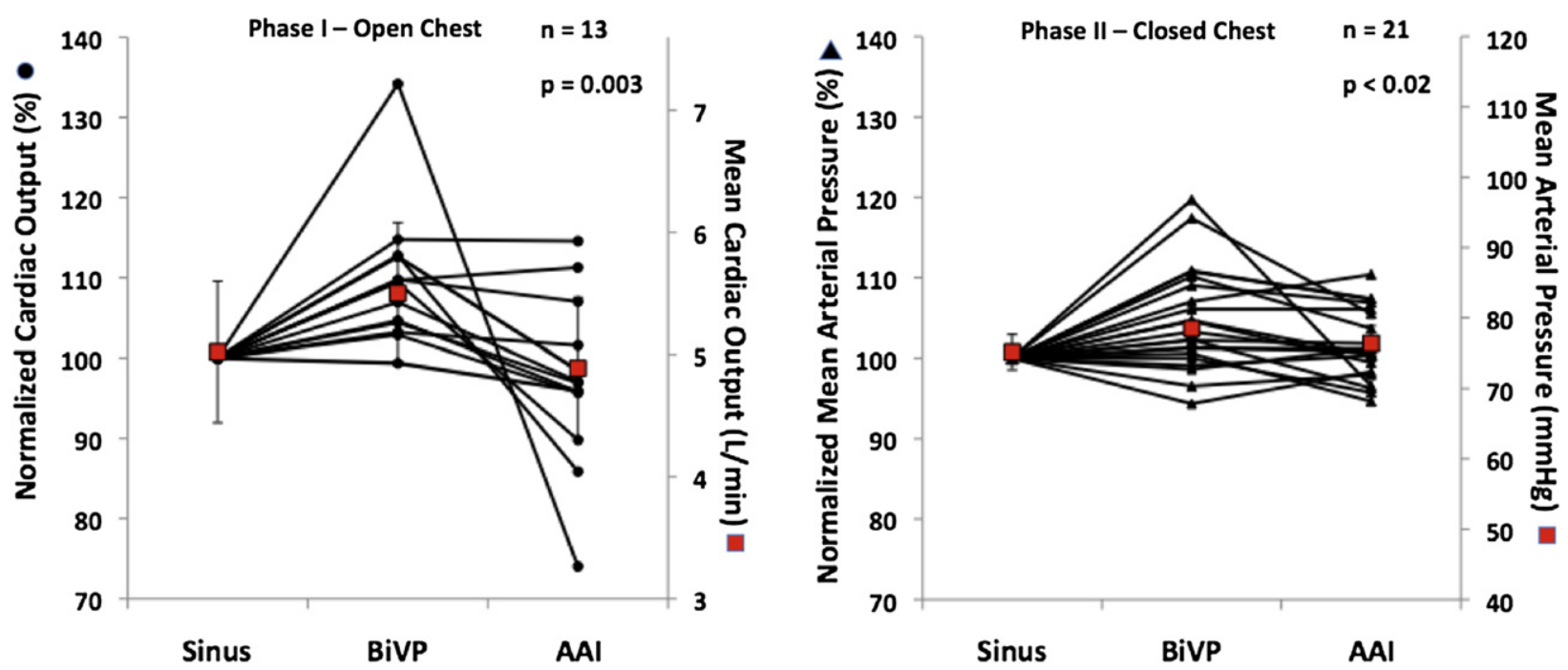

FIGURE 6. Right, Paired plots demonstrate effects of sinus rhythm, optimized biventricular pacing (BiVP), and atrial pacing (AAI) on normalized mean arterial pressure $(M A P)$ for individual patients in present study. Data compared with data for effects on cardiac output of phase I from previous substudy of this clinical trial. Square symbols indicate averages with standard errors. Changes were statistically significant. 
for whom the default $0 \mathrm{~ms}$ VVD was least favorable, the optimal VVD was $60 \mathrm{~ms}$ or greater, suggesting conduction delays that necessitate optimization. The practical limitations on the number of parameters that can be tested prevented simultaneous variation in AVD and VVD using this protocol; however, these issues will be addressed in the analysis of data from phase III.

Consistent with our previous study of phase $\mathrm{I},{ }^{15}$ we found intrinsic benefit in BiVP, independent of the heart rate during skin closure. Optimized BiVP increased the MAP by $3 \%$ and $5 \%$ compared with AAI and NSR, respectively. This increase in the MAP compared with the NSR was predominantly accounted for by the $6 \%$ increase in diastolic blood pressure. Because the testing intervals were brief and the inotropes and vasoconstrictors were held constant, it seems unlikely that changes in systemic vascular resistance were an important source of error. ${ }^{21}$ Compared with AAI, however, the increase in the MAP had contributions from the diastolic and systolic pressures both, likely owing to an increased pulse pressure (and thus stroke volume) with BiVP. BiVP appears to correct the detriment to pulse pressure of pacing at a greater heart rate while concurrently improving the diastolic pressure.

In our previous study, the hemodynamics of NSR and AAI were qualitatively similar, and the CO with AAI was actually (insignificantly) less than with NSR. In the present study, the MAP with AAI was intermediate between that of NSR and BiVP, suggesting a trend toward increasing importance of AAI (Figure 6). This finding is consistent with previous work by Eberhardt and colleagues ${ }^{13}$ and by previous findings from our own laboratory that suggest the effects of $\mathrm{BiVP}$ and AAI are roughly equivalent on the first postoperative day. It therefore appears that the benefits of BiVP on contractility are most important in the early post-CPB period, decreasing in importance with time as the ischemiareperfusion injury resolves.

Is the small change in MAP observed in our study clinically important? Increasing the MAP could potentially affect postoperative mortality, stroke, and cardiac complications. ${ }^{24}$ The MAP has previously been shown to correlate positively with the $\mathrm{CO}$, often underestimating increases in $\mathrm{CO} .{ }^{21}$ Our experience in comparing the hemodynamic benefits has been that the percentage of changes in the MAP are roughly one half those measured by $\mathrm{CO}$ when both indexes are used simultaneously. For example, we recently reported a $10 \%$ increase in $\mathrm{CO}$ when BiVP was compared with NSR; the corresponding increase in the MAP was $5 \% .{ }^{15}$ If the $4 \%$ improvement in the MAP for BiVP versus NSR reported in the present study corresponds to an $8 \%$ increase in $\mathrm{CO}$, as we believe, the improvement in $\mathrm{CO}$ after chest closure would be similar to that previously reported in phase I.

We have not yet determined whether reoptimization after chest closure is necessary or if the phase I protocol would be adequate for overnight pacing. Given the dynamic early period of cardiac recovery from ischemia and reperfusion, reoptimization during skin closure might prove essential. Although this trial was directed at optimization of $\mathrm{CO}$, optimization in phase II, using the MAP, has emerged as most important in the clinical outcome of this trial. Phase II optimization commonly changes the pacing parameters defined in phase I and remained in effect in the BiVP group for 12 to 24 hours during the first postoperative night. In contrast, the phase I protocol is used for 1 to 2 hours, and the phase III protocol is not used at all for extended pacing. Nevertheless, preliminary data have demonstrated the clinical benefits of the phase II protocol for both the vasoconstrictor inotrope score and urine output during the first postoperative night in the BiVP group compared with the standard of care group. ${ }^{25}$

The BiPACS protocol requires 206 completed studies for sufficient statistical power to demonstrate a $15 \%$ benefit in thermal dilution $\mathrm{CO}$ for the BiVP group. We have been exceptionally fortunate to have received National Institutes of Health R01 grant support for the present study. Given the current realities of National Institutes of Health funding, however, the trial might have to be terminated before reaching its ultimate goal. Premature termination of the BiPACS trial will make the determination of secondary end points problematic, including patient selection, relevance of transesophageal echocardiography for defining the optimal candidates, importance of the pacing site, and the effects on morbidity, mortality, and hospital costs.

\section{CONCLUSIONS}

Optimization of temporary BiVP after chest closure increases the MAP in selected patients. Optimization of both AVD and VVD contribute to the benefit, and optimized BiVP is superior to AAI or sinus rhythm. Optimization should be performed routinely when using temporary BiVP, and determination of the arterial pressure is a promising, less-invasive, technique. In view of the broad scatter of the optimum AVD, individual optimization is warranted, in preference to default values.

The authors thank the BiPACS trial patients and investigators and the Columbia University Division of Cardiothoracic Surgery for their participation. Alexandra Murata, our Study Coordinator for 2010-2011 provided important assistance with manuscript preparation.

\section{References}

1. Hunt SA, Abraham WT, Chin MH, Feldman AM, Francis GS, Ganiats TG, et al. 2009 focused update incorporated into the ACC/AHA 2005 Guidelines for the Diagnosis and Management of Heart Failure in Adults: a report of the American College of Cardiology Foundation/American Heart Association Task Force on Practice Guidelines: developed in collaboration with the International Society for Heart and Lung Transplantation. Circulation. 2009;119:e391-479.

2. Young JB, Abraham WT, Smith AL, Leon AR, Lieberman R, Wilkoff B, et al Multicenter InSync ICD Randomized Clinical Evaluation (MIRACLE ICD) 
Trial Investigators. Combined cardiac resynchronization and implantable cardioversion defibrillation in advanced chronic heart failure: the MIRACLE ICD Trial. JAMA. 2003;289:2685-94.

3. Abraham WT, Fisher WG, Smith AL, Delurgio DB, Leon AR, Loh E, et al. Cardiac resynchronization in chronic heart failure. N Engl J Med. 2002;346: 1845-53.

4. León AR, Abraham WT, Brozena S, Daubert JP, Fisher WG, Gurley JC, et al. InSync III Clinical Study Investigators. Cardiac resynchronization with sequential biventricular pacing for the treatment of moderate-to-severe heart failure. $\mathrm{J} \mathrm{Am}$ Coll Cardiol. 2005;46:2298-304.

5. Steendijk P, Tulner SA, Bax JJ, Oemrawsingh PV, Bleeker GB, van Erven L, et al. Hemodynamic effects of long-term cardiac resynchronization therapy: analysis by pressure-volume loops. Circulation. 2006;113:1295-304.

6. Moss AJ, Hall WJ, Cannom DS, Klein H, Brown MW, Daubert JP, et al. Cardiacresynchronization therapy for the prevention of heart-failure events. $N$ Engl $J$ Med. 2009;361:1329-38.

7. Reynolds CR, Gold MR. Cardiac resynchronization therapy in mild heart failure: a review of the REVERSE and MADIT-CRT trials. Curr Cardiol Rep. 2010;12: 367-73.

8. Whinnett ZI, Davies JE, Willson K, Chow AW, Foale RA, Davies DW, et al. Determination of optimal atrioventricular delay for cardiac resynchronization therapy using acute non-invasive blood pressure. Europace. 2006;8:358-66.

9. De Lurgio D, Boehmer J, Higgins S, De Marco T, Guidici M, Hoyhtya A, et al. Simultaneous biventricular pacing with optimized atrioventricular delay results in more reverse remodeling versus other resynchronization modalities in DECREASE-HF. J Card Fail. 2007;13(Suppl 2):S141.

10. Marsan NA, Bleeker GB, Van Bommel RJ, Borleffs CJ, Bertini M, Holman ER, et al. Cardiac resynchronization therapy in patients with ischemic versus nonischemic heart failure: differential effect of optimizing interventricular pacing interval. Am Heart J. 2009;158:769-76.

11. Yau TM, Fedak PW, Weisel RD, Teng C, Ivanov J. Predictors of operative risk for coronary bypass operations in patients with left ventricular dysfunction. J Thorac Cardiovasc Surg. 1999;118:1006-13.

12. Antonio BL, Royster RL, Kon ND, Zvara DA. Acute biventricular pacing after cardiopulmonary bypass decreases myocardial dyssynchrony and increases cardiac index. J Cardiothorac Vasc Anesth. 2007;21:570-1.

13. Eberhardt F, Heringlake M, Massalme MS, Dyllus A, Misfeld M, Sievers HH, et al. The effect of biventricular pacing after coronary artery bypass grafting: a prospective randomized trial of different pacing modes in patients with reduced left ventricular function. J Thorac Cardiovasc Surg. 2009;137:1461-7.
14. Nelson GS, Berger RD, Fetics BJ, Talbot M, Spinelli JC, Hare JM, et al. Left ventricular or biventricular pacing improves cardiac function at diminished energy cost in patients with dilated cardiomyopathy and left bundle-branch block. Circulation. 2000;102:3053-9.

15. Wang DY, Richmond ME, Quinn TA, Mirani AJ, Rusanov A, Yalamanchi V, et al. Optimized temporary biventricular pacing acutely improves intraoperative cardiac output after weaning from cardiopulmonary bypass: a substudy of a randomized clinical trial. J Thorac Cardiovasc Surg. 2011;141:1002-8.

16. Rabkin DG, Cabreriza SE, Curtis LJ, Mazer SP, Kanter JP, Weinberg AD, et al. Load dependence of cardiac output in biventricular pacing: right ventricular pressure overload in pigs. J Thorac Cardiovasc Surg. 2004;127:1713-22.

17. Berberian G, Quinn TA, Kanter JP, Curtis LJ, Cabreriza SE, Weinberg AD, et al. Optimized biventricular pacing in atrioventricular block after cardiac surgery. Ann Thorac Surg. 2005;80:870-5.

18. O’Donnell D, Nadurata V, Hamer A, Kertes P, Mohamed U. Long-term variations in optimal programming of cardiac resynchronization therapy devices. Pacing Clin Electrophysiol. 2005;28(Suppl 1):S24-6.

19. Porciani MC, Dondina C, Macioce R, Demarchi G, Cappelli F, Lilli A, et al. Temporal variation in optimal atrioventricular and interventricular delay during cardiac resynchronization therapy. J Card Fail. 2006;12:715-9.

20. Brecker SJ, Xiao HB, Sparrow J, Gibson DG. Effects of dual-chamber pacing with short atrioventricular delay in dilated cardiomyopathy. Lancet. 1992;340: 1308-12.

21. Prasso JE, Berberian G, Cabreriza SE, Quinn TA, Curtis LJ, Rabkin DG, et al. Validation of mean arterial pressure as an indicator of acute changes in cardiac output. ASAIO J. 2005;51:22.

22. Quinn TA, Cabreriza SE, Richmond ME, Weinberg AD, Holmes JW, Spotnitz HM. Simultaneous variation of ventricular pacing site and timing with biventricular pacing during acute ventricular failure improves function by interventricular assist. Am J Physiol Heart Circ Physiol. 2009;297:H2220-6.

23. Berberian G, Cabreriza SE, Quinn TA, Garofalo CA, Spotnitz HM. Left ventricular pacing site-timing optimization during biventricular pacing using a multielectrode patch. Ann Thorac Surg. 2006;82:2292-4.

24. Gold JP, Charlson ME, Williams-Russo P, Szatrowski TP, Peterson JC, Pirraglia PA, et al. Improvement of outcomes after coronary artery bypass: a randomized trial comparing intraoperative high versus low mean arterial pressure. J Thorac Cardiovasc Surg. 1995;110:1302-11.

25. Nguyen HV, Havalad V, Aponte-Patel L, Murata A, Wang DY, Rusanov A, et al. Temporary biventricular pacing decreases the vasoactive-inotropic score after cardiac surgery: a substudy of a randomized clinical trial. J Thorac Cardiovasc Surg. July 28, 2012 [Epub ahead of print]. 